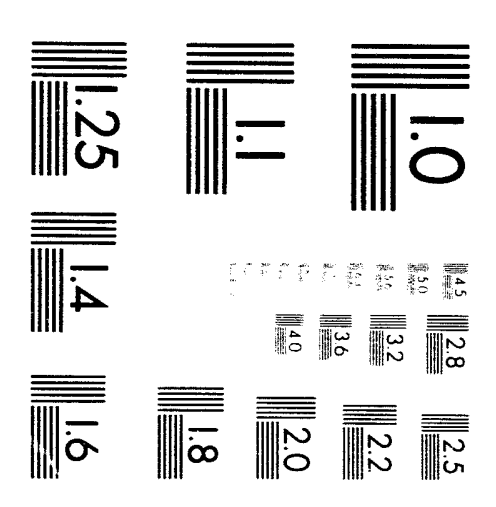



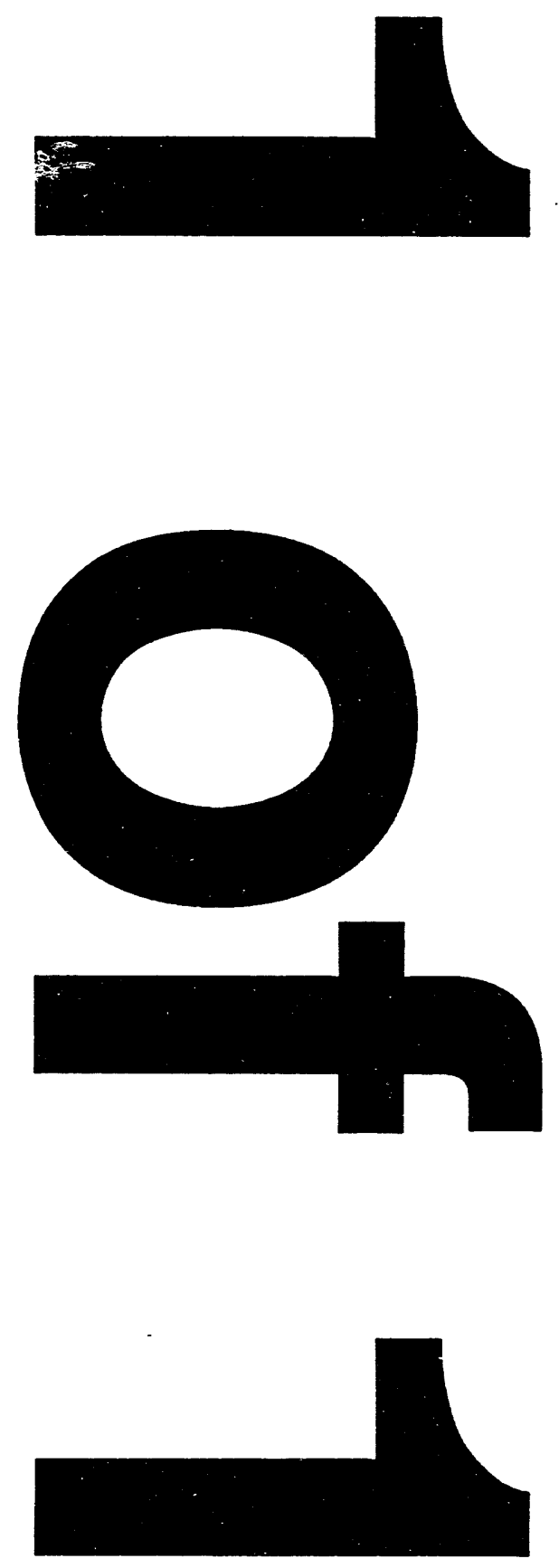


\title{
Using the TSAR Electromagnetic Modeling System
}

\author{
Steven T. Pennock and Gary W. Laguna
}

September 1993

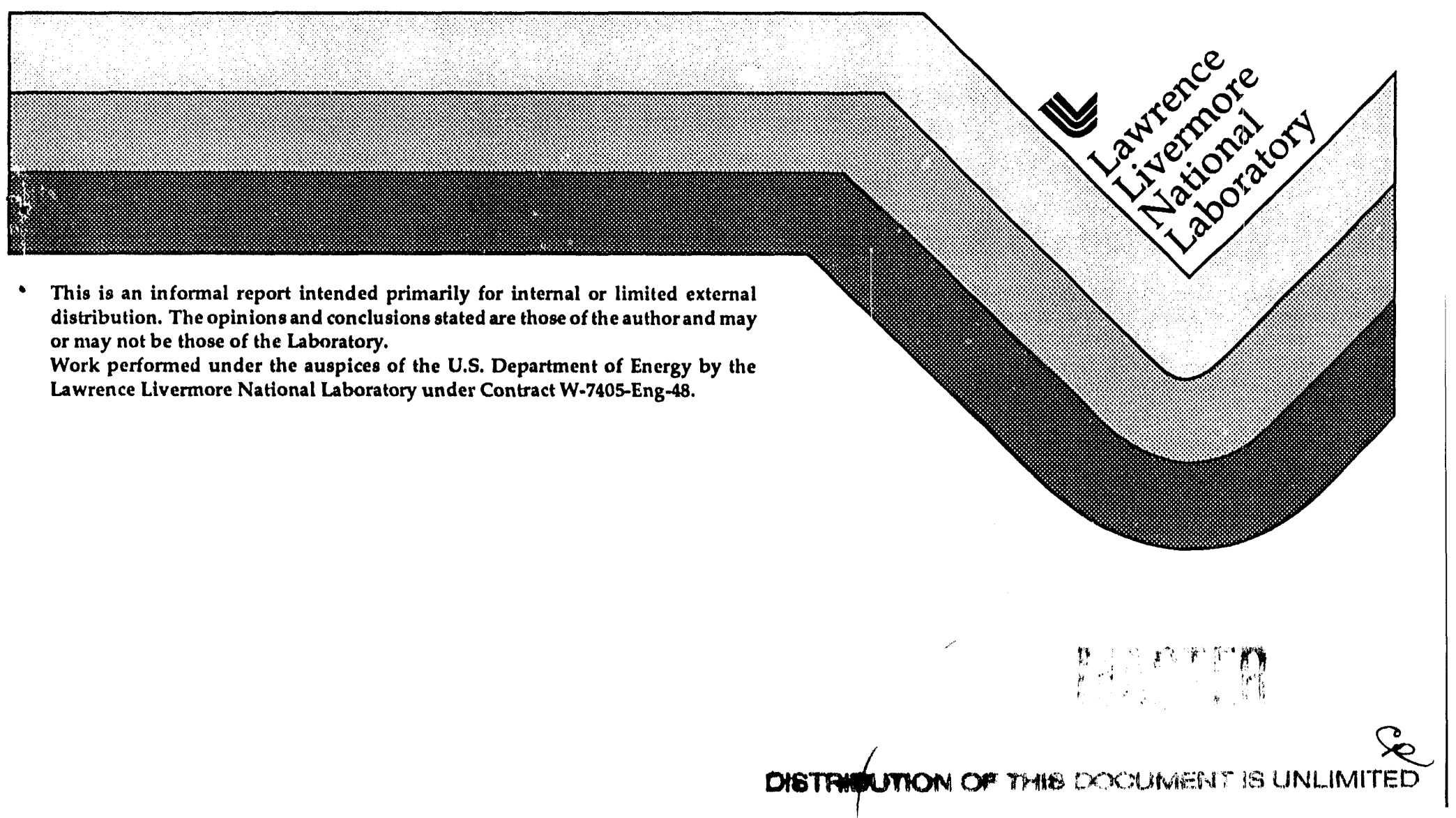




\section{DISCLAIMER}

This document was prepared as an account of work sponsored by an agency of the United States Government. Neither the United States Government nor the University of California nor any of their employees, makes any warranty, express or implied, or assumes any legal liability or responsibility for the accuracy, completeness, or usefulness of any information, apparatus, product, or process disclosed, or represents that its use would not infringe privately owned riphts. Reference herein to any specific commercial products, process, or service by trade nanie, trademark, manufacturer, or otherwise, does not necessarily constitute or imply its endorsement, recommendation, or favoring by the United States Government or the University of California. The views and opinions of authors expressed herein do not necessarily state or reflect those of the United States Government or the Univer sity of California, and shall not be used for ad vertising or product endorsement purposes.

This report has been reproduced

directly from the best available copy.

Available to DOE and DOE contractors from the

Office of Scientific and Technical Information

P.O. Box 62, Oak Ridge, TN 37831

Prices a vailable from (615) 576.8401, FTS 626.8401

A vailable to the public from the

National Technical Information Service

U.S. Department of Commerce

5285 Port Royal Rd.,

Springfield, VA 22161 


\title{
Using the TSAR Electromagnetic Modeling System
}

\author{
Steven T. Pennock and Gary W. Laguna \\ Lawrence Livermore National Laboratory \\ P.O. Box 808, L--156, Livermore, CA 94550
}

\section{Chapter 1}

\section{INTRODUCTION}

A new user, upon receipt of the TSAR EM modeling system, may be overwhelmed by the number of software packages to learn and the number of manuals associated with those packages. This is a document to describe the creation of a simple TSAR model, beginning with an MGED solid and continuing the process through final results from TSAR. It is not intended to be a complete description of all the parts of the TSAR package. Rather, it is intended simply to touch on all the steps in the modeling process and to take a new user through the system from start to finish.

There are six basic parts to the TSAR package. The first, MGED, is part of the BRLCAD package and is used to rreate a solid model. The second part, ANASTASIA, is the program used to sample the solid model and create a finite--difference mesh. The third program, IMAGE, lets the user view the mesh itself and verify its accuracy. If everything about the mesh is correct, the process continues to the fourth step, SETUP_TSAR, which creates the parameter files for compiling TSAR and the input file for running a particular simulation. The fifth step is actually running TSAR, the field modeling program. Finally, the output from TSAR is placed into SIG, B2RAS or another program for postprocessing and plotting. Each of these steps will be described below.

The best way to learn to use the TSAR software is to actually create and run a simple test problem. As an example of how to use the TSAR package, let's create a sphere with a rectangular internal cavity, with conical and cylindrical penetrations connecting the outside to the inside, and find the electric field inside the cavity when the object is exposed to a Gaussian plane wave. We will begin with the solid modeling software, MGED, a part of the BRL-CAD modeling release. 


\section{Chapter 2}

CREATING A SOLID MODEL.

If the BRL, package has been installed properly, (installation instructions are included with the BRL-CAD software), one begins by typing:

\section{mged hollow sphere.g,}

where hollow_sphere.g is the filename chosen lor the MGED database. Below is an example MGED session, with responses to prompts in bold face.

BRL-CAD Release 4.0 Graphics Editor (MGED)

Wed Nov 13 15:58:13 PST 1991, Compilation 11

laguna@ wichita.Ilnl.gov:/public I/BRL4.0/BRL4.0/.mged.ibm

hollow_sphere.g: A file or directory in the path name does not exist.

Create new database $(y \mid n)[n]$ ? y attach (nultekltek4109lps/plotlsgilX) [nul'? sgi

(Choose the appropriate device driver for your workstation.)

ATTACHING sgi (SGI 4d)

Untitled MGED Database (units=mm)

Begin by building a sphere with a one meter radius, located at the origin. Units are in millimeters, although they could be in meters, centimeters, feet or inches. The command to charge the default units is units ab, where ab is $\mathbf{m m}, \mathbf{c m}, \mathbf{m}$, in or ft. We will name the primitive sphere.s. The naming convention calls for solids to end with a.$s$ extension and regions, (to be introduced later), with an rextension, for easy identification.

mged $>$ in sphere.s sph

Enter $X, Y, Z$ of vertex: 000

Enter radius: 1000

51 vectors in $0 \mathrm{sec}$

Now enter a rectangular solid inside the sphere.

mged $>$ in box $1 . s$ arb8

Enter $X, Y, Z$ for point $1:-\mathbf{- 3 5 0}-\mathbf{1 5 0} \quad \mathbf{4 0 0}$

Enter $X, Y, Z$ for point 2: $350 \quad 150-400$

Enter $X, Y, Z$ for point 3: $350 \quad 350-400$

Enter $X, Y, Z$ for point 4: $\mathbf{- 3 5 0} 350-\mathbf{4 0 0}$

Enter $X, Y, Z$ for point 5: $\mathbf{- 3 5 0}-\mathbf{1 5 0} 200$

Enter X, Y, Z for point 6: $350-150200$

Enter $X, Y, Z$ for point 7: 350 350 200

Enter X, Y, Z for point 8: -350 350200

16 vectors in $0 \mathrm{sec}$

The arb8 command is for an eight-sided solid of arbitrary shape. An easier method for a rectangular solid is the box command. Another possibility would be to enter a simple box of arbitrary size using the make command, then adjust the box using the solid editor. 
mged $>$ in box 2.5 box

Enter $X, Y, Z$ of vertex: $\mathbf{- 7 4 0}-\mathbf{- 5 3 0}-\mathbf{6 4 0}$

Enter $X, Y, Z$ of vector $H: 001240$

Enter X, Y, Z of vector W: 010300

Enter $X, Y, Z$ of vector D: 144000

16 vectors in $0 \mathrm{sec}$

Now enter a cylinder at an angle to the box.

mged $>$ in cyll.s rec

Enter $X, Y, Z$ of vertex: 0 -515 -1060

Enter X, Y, Z of height $(H)$ vector: 011602000

Enter radius: 100

42 vectors in $0 \mathrm{sec}$

Finally, enter an elliptical cone through the sphere and box.

mged $>$ in cone $1 . s$ tgc

Enter X, Y, Z of vertex: $\mathbf{- 1 3 0 0} \mathbf{1 0 2} \mathbf{- 9 3}$

Enter $X, Y, Z$ of height $(\mathrm{H})$ vector: 260000

Enter X, Y, Z of vector A: 02300

Enter X, Y, Z of vector B: 00128

Enter scalar c: $\mathbf{1 3 2 . 5}$

Enter scalar d: 69.5

42 vectors in $1 \mathrm{sec}$

Use the command tops to look at what objects exist at the top level.

mged $>$ tops

box l.s conel.s sphere.s

box 2.5 cyll.s

Currently there are five primitive solids, two of which are boxes, and all are located in the same spatial region. (As noted earlier, the .s extension signifies a solid). To get rid of one box, use the kill command. We will eliminate the smaller box so that more of the sphere will be hollow. This will mean fewer nodes to be displayed when we look at the mesh.

mged > kill box1.s

Now the relationship between the solids must be defined by creating a region. This consists of a name for the region and the boolean operations that must be performed between solids to create the region. MGED allows no explicit grouping of Boolean operators nor is there an operator precedence. Instead, there is an implicit grouping of operations between union operators. See appendix B of the Anastasia manual for further details.

MGED allows unions (u), subtractions (-), and intersections (+) to be performed. In this case, one would simply subtract the box, cone and cylinder from the sphere to obtain the hollow sphere with penetrations. We will call this region carved_sphere.r.

mged $>\mathbf{r}$ carved_sphere.r u sphere.s - box2.s - cone1.s - cyl1.s

Defaulting item number to 1001 
Creating region $\mathrm{i} d=10(0)$, air $=0, \operatorname{los}=100)$, (ilFThaterial $=1$

To make things more interesting, let's also create a simple solid that just consists of the cylinder. This will allow us to assign a different dielectric constant to this region.

mged $>\mathbf{r}$ cylinder.r u cyll.s

Defaulting item number to 1002

Creating region $i d=1001$, air $=0,10 s=100$, GIFTmaterial $=1$

It is very useful in MGED to be able to display regions using different colors. A default color table can be created, then regions can be assigned item numbers to correspond to certain colors. We will create a simple two-color table and assign one color to each of our regions. Each line of the color table contains the lowest and highest item numbers for each color, then the red, green and blue values for that color, then finally a short descriptive string for each color.

mged> color 10012502550 green

mged> color 12615025500 red

Now assign each of our regions to a particular color using the item command.

mged $>$ item carved_sphere.r 105

mged $>$ item cylinder.r 130

Clearing the display with the $\mathrm{Z}$ command, then displaying the regions should reflect the changes made to the colors.

mged $>\mathbf{Z}$

mged $>$ E carved_sphere.r cylinder.r

One final step must be done in MGED before proceeding to build a mesh. We need to assign a finite-difference material to each of the regions. Later we will assign constituitive parameters to each of these materials. The finite-difference materials are entered after the Parameter String prompt, and have the form fd_mat=num, where num is number between 1 and 16. The Material prompt is asking for a material definition for the ray tracer, and seems to recognize plastic, mirror and glass. The color prompt allows rgb values to be entered, and the color will show up in any ray traces done with this database.

We generally reserve finite-difference material number 1 for the background material, which by default is free space. You might want to follow the same practice and only change the background material parameters if the object sits in a material other than vacuum. (Use care if the background material is changed. The velocity of propagation and the boundary conditions are only two things that might need to be changed). Use numbers 2 through 16 for the model's materials.

mged $>$ mater carved_sphere.r

Material $=$

Material? ('del' to delete, CR to skip) plastic

Param $=$

Parameter string? ('del' to delete, CR to skip) $\mathbf{f d}$ _mat $=\mathbf{8}$

Color $=$ (No color specified $)$

Color R G B (0..255)? ('del' to delete, CR to skip) 02550

Inherit $=0$ : lower nodes (towards leaves) override

Inheritance (0l1)? (CR to skip) [CR] 
mged $>$

mged $>$ mater cylinder.r

Material =

Matcrial? ('del' to delete, CR to skip) plastic

Param =

Parameter string? ('del' to delete, CR to skip) fd mat=3

Color $=($ No color specified $)$

Color R G B (0..255)? ('del' to delete, CR to skip) 255 () 0

Inherit $=0$ : lower nodes (towards leaves) override

Inheritance (0|I)? (CR to skip) [CR]

At this point, if we are satisfied by the way the solid model looks, we can leave mged. Before we do, though, we might want to use the saveview command to create a file which will raytrace the object.

To use the saveview command, first display the model on the screen exactly as you would like it to appear in the raytrace. You can use the slide is to rotate the model to the desired position, or use the vrot command. Once you have the model in the position you would like, type:

\section{saveview filename $-\mathrm{s} \mathbf{m}-\mathrm{C \# / \# / \#}$}

This will create an executable named 'filename' which can be run to generate a raytraced view of the object. The argument $-\mathrm{s} m$ will produce a picture $\mathrm{m}$ pixels by $\mathrm{m}$

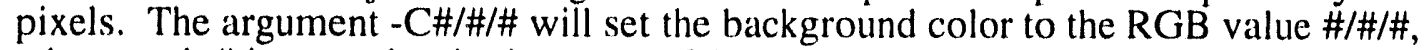
where each \# is a number in the range of 0 to 255 . For example, entering:

\section{saveview picture1 -s $256-C 140 / 190 / 140$}

would create an executable file picture 1. Leave MGED by typing ' $q$ ' after the prompt:

mged $>$ q

To produce a ray-traced view of the solid model, simply type the name of the saveview file. Since ray-tracing may take a very long time, it is best to lower the priority with the nice command and run it in the background:

\section{nice picture1 \&}

This command will produce a ray-traced picture, picturel.pix, which will have a resolution of 256 pixels by 256 pixels and a pale green background.

To display the picture, first convert to ras format with the command:

\section{pix2ras -s 256 picture1.pix picture1.ras}

then display the picture created by typing:

\section{xtras picture1.ras.}

To close the display, place the pointer inside the display window and type q for quit. 


\section{Chapter 3}

\section{MAKING A FINITE DIFFERENCE MESH}

Once we have left MGED, the next step is to use ANA to create a finite difference mesh, based on the solid model just created. To do this, type:

\section{ana hollow_sphere.g}

Anastasia offers the choice of either a graphical button menu for command entry, or keyboard entry using the teletype mode. The following is a session using teletype mode. In graphical mode, mouse clicks would be used for selecting, and keyboard entry would only occur when prompted for specific input.

Select Display Type ( graphics I tty ) tty

Anastasia Version 1.13 -- April 1992

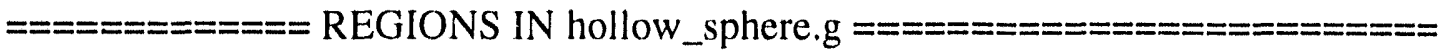

cylinder.r

carved_sphere.r cr

\section{SELECT AND GROUP REGIONS TO BE MESHED}

Each region of the solid model that is to be meshed must be assigned to a group. Each group has its own set of mesh parameters and is meshed separately. After a group is meshed, that mesh will be merged with the mesh(es) of previous groups. Group 1 has been created for you.

Regions are added to groups by using the commands below.

$$
\begin{aligned}
& \text { a --- add or delete regions for group [1] } \\
& \text { c--- create new group } \\
& \text { g --- list all group assignments } \\
& \mathrm{r} \text {--- list all regions } \\
& \mathrm{s} \text {--- select alternative group } \\
& \mathrm{x} \text {--- accept group assignments }
\end{aligned}
$$

Enter command: a

\section{ADD REGIONS TO GROUP NUMBER [1]}

Enter names of regions to be added, one region per line. Regular expressions are acceptable. Terminate with a ': on a line by itself.

For this simple model, add all the regions to one group. More complicated models may require more than one group.

\section{cylinder.r}

adding [cylinder.r]

carved_sphere.r

adding [carved_sphere.r] 


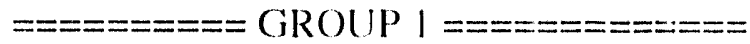

cylinder.r

carved_sphere.r

$$
\begin{array}{ll}
\text { a - } & \text { add or delete regions for group [1] } \\
\text { - } & \text { create new group } \\
\text { g- } & \text { list all group assignments } \\
\text { r - } & \text { list all regions } \\
\text { s - } & \text { select alternative group } \\
\text { x - } & \text { accept group assigmments }
\end{array}
$$

Enter command: $x$

Accept the group assignments and move to the next step, selecting parameters for this particular mesh.

$==========$ GROUP |

cylinder.r

carved_sphere.r

EXTENT OF BOUNDING RPP IN MILLIMETERS

$\begin{array}{ccc} & \text { MIN } & \text { MAX } \\ X & -1000.00 & 1000.00 \\ Y & -1000.00 & 1000.00 \\ Z & -1110.17 & 1000.00\end{array}$

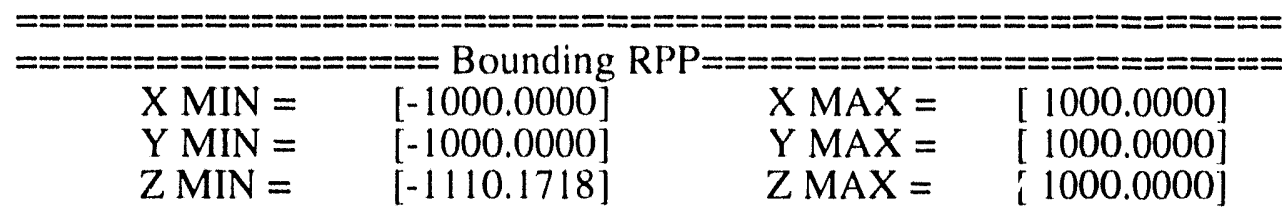

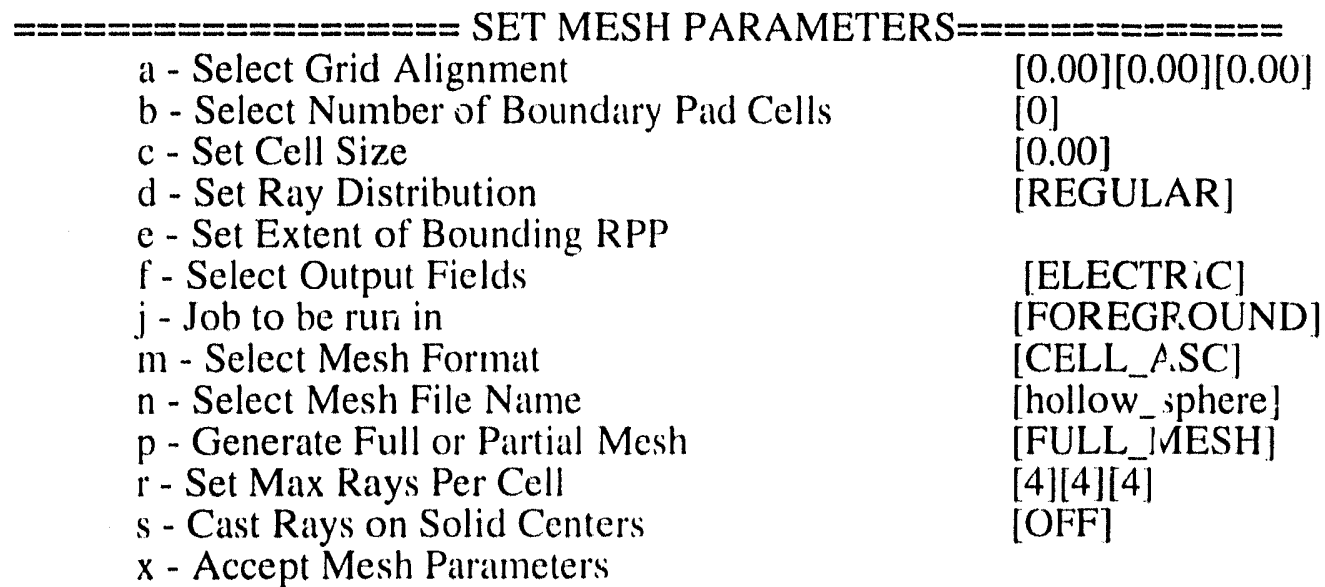

Enter Command: b 


\section{SELECT THE NUMBER (OF BOUNDARY PAD) CELISS}

Determines the number of emply cells that are to be added to the boundaries of the model.

We need to surround the model with some free space cells, so that the radiation boundary conditions and near/far-field interface operate correctly. Standard practice is to allow 8 cells from the problem space boundary to the model. More maly be necessary to avoid reflections from the corners of the problem space.

Enter integer number of pad cells: 8

Number of pad cells set to $|8|$

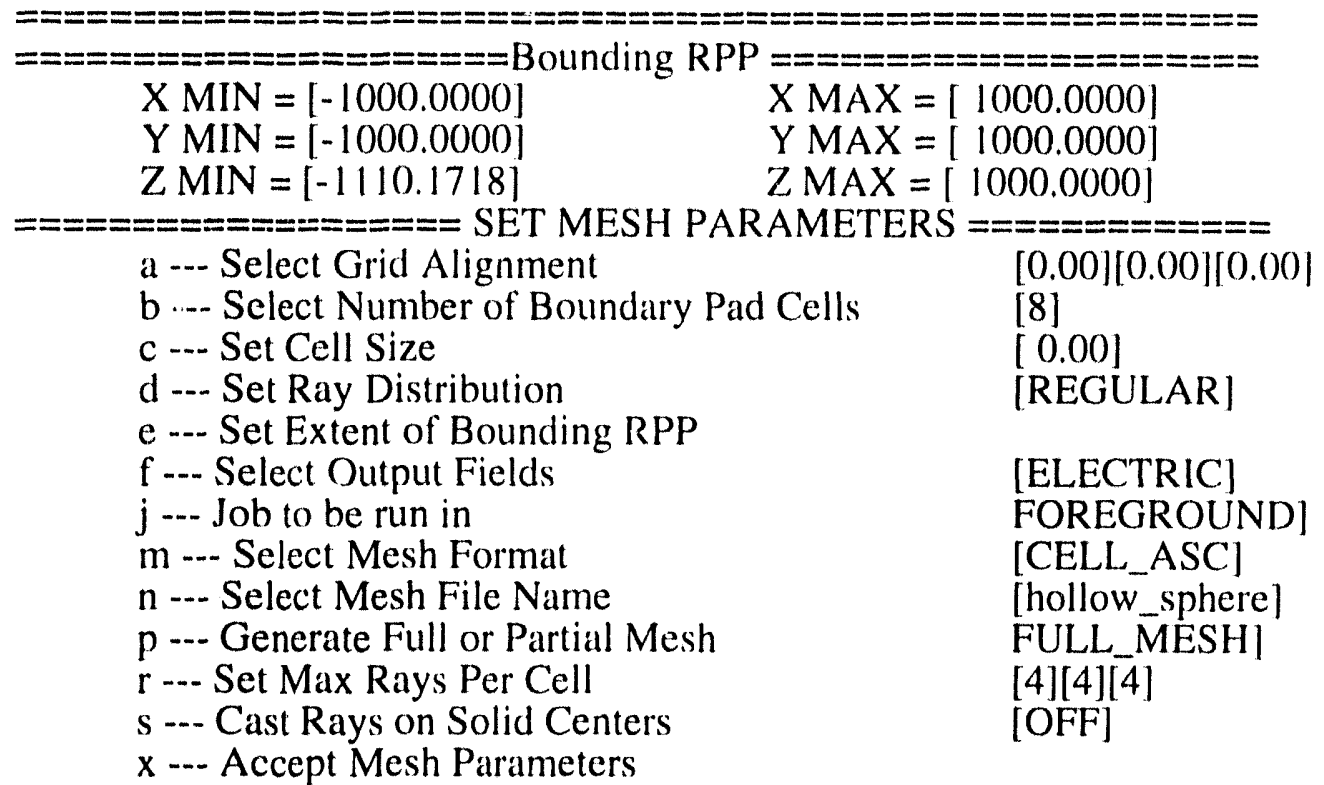

Enter Command: c

SET GRID CELL SIZE

Select cell size for the mesh in millimeters

Set the cell size based on the highest frequency of interest. 10 cells per wavelength is the standard value.

Enter Cell Size [mm] : 50

Cell size set to $[50.00] \mathrm{mm}$

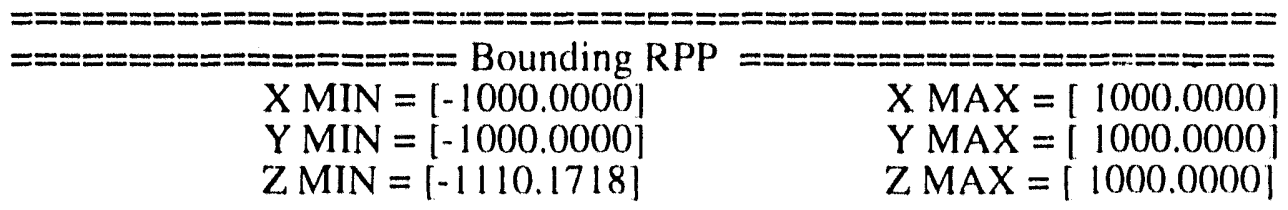




\begin{tabular}{|c|c|}
\hline a -.. Select Number of Boundary Pad cells & {$[0.00|| 0.000|| 0.00]$} \\
\hline b -.. Select Number of Boundary Pad cells & $|8|$ \\
\hline c -.. Set Coll Siza & {$[50.00]$} \\
\hline d .... Set Ray Distribution & |REGULAR| \\
\hline c.-. Set Exient of Bounding RPP & \\
\hline I -.- Select Output Ficlds & |ELECTRIC| \\
\hline j -.. Job to be ruin in & |FOREGROUND| \\
\hline m --. Select Mesh Formall & [CELL_ASC] \\
\hline n -.- Select Mesh File Name & [hollow_sphere] \\
\hline p -.- Generate Full or Partial Mesh & [FULL_MESH] \\
\hline r --- Set Max Rays Per Cell & {$[4][4][4]$} \\
\hline$s$-. Cast Rays on Solid Centers & {$[\mathrm{OFF}]$} \\
\hline x -.- Accept Mesh Parameters & \\
\hline
\end{tabular}

Change the ray distribution from regular to random. This isn't really necessary, it is just included as an example of a parameter that can be toggled between values.

Enter Command: d

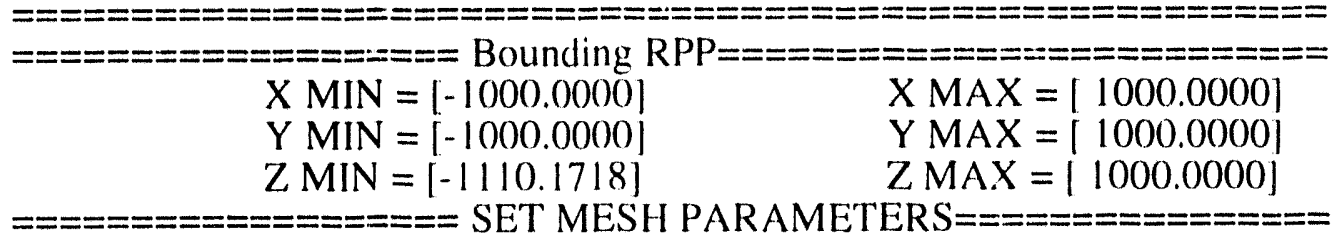

a -.- Select Grid Alignment

b -.. Select Number of Boundary Pad Cells

c -.. Set Cell Size

d -..- Set Ray Distribution

e -.- Set Extent of Bounding RPP

f -.. Select Output Fields

j --- Job to be run in

m -.- Select Mesh Format

n -.. Select Mesh File Name

p -.. Generate Full or Partial Mesh

r -.- Set Max Rays Per Cell

$s$-- Cast Rays on Solid Centers

x -.. Accept Mesh Parameters
[0.00] [0.00] [0.00]

[8]

[ 0.00$]$

[RANDOM]

[ELECTRIC]

[FOREGROUND]

CELL_ASC]

[hollow_sphere]

[FULL_MESH]

[4][4][4]

$[\mathrm{OFF}]$

Enter Command: $\mathbf{n}$

Shorten the output file name, as ANA will append 6 characters to the specified name.

SELECT A BASE FILE NAME FOR THE GENERATED MESHES

Enter a name for the output mesh file: sphere 


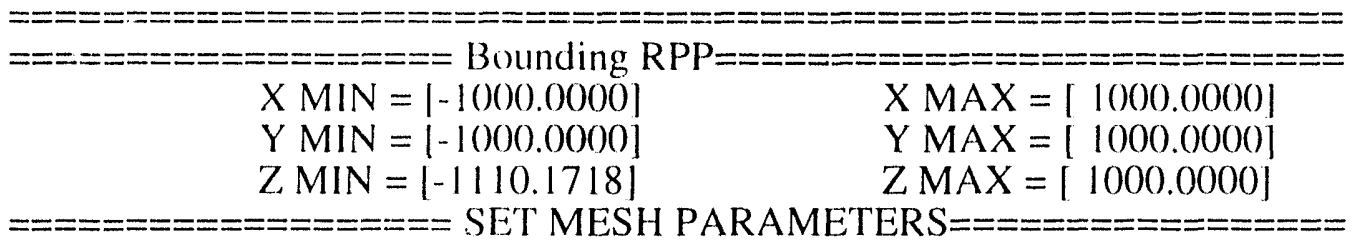

a --- Select Grid Alignment

b --- Select Number of Boundary Pad Cells

c --- Set Cell Size

d --- Set Ray Distribution

e --- Set Extent of Bounding RPP

f -.. Select Output Fields

$\mathrm{j}$--- Job to be run in

m --- Select Mesh Format

n --- Select Mesh File Name

p -.- Generate Full or Partial Mesh

r --- Set Max Kays Per Cell

$s$--- Cast Rayss on Solid Centers

x --- Accept Mesh Parameters
$[0.00][0.00)[0.00]$

[8]

[0.00]

[REGULAR

[ELECTRIC]

[FOREGROUND]

[CELL_ASC]

[sphere]

[FULL_MESH]

[4][4][4]

[OFF]

Enter Command: $\mathbf{r}$

\section{SET THE MAXIMUM NUMBER OF RAYS PER OCTANT}

Sets the maximum number of rays that will be generated for any octant to determine whether that octant is in or out of the solid. Fewer rays will generally result in certain octants being incorrectly classified whereas more rays require more computer time.

Set the maximum number of rays cast in each direction based on the model being sampled. Parts which are thin in the $y$-direction may require $y$-rays to be cast in order to be found. Y-rays are the most time-consuming, though, so should be used only when necessary.

Enter the maximum number of rays per octant in each direction $(x, y, z): 404$ Maximum rays per octant set to [4][0][4]

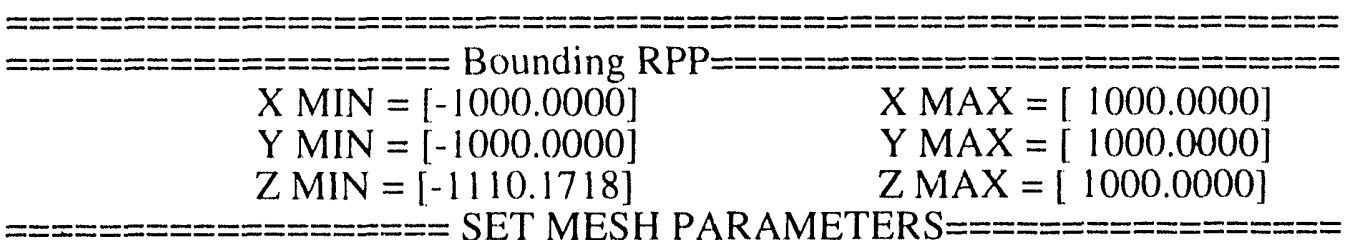

a --- Select Grid Alignment

b --- Select Number of Boundary Pad Cells

c -.- Set Cell Size

d -.. Set Ray Distribution

e --- Set Extent of Bounding RPP

f --- Select Output Fields

j--- Job to be run in

$\mathrm{m}$--. Select Mesh Format

n --- Select Mesh File Name
$[0.00][0.00][0.00]$

[8]

[0.00]

[REGULAR]

[ELECTRIC]

[FOREGROUND]

[CELL_ASC]

[hollow_sphere] 
p --- Generate Full or Partial Mesh

r -.- Set Max Rays Per Cell

s -.- Cast Rays on Solid Centers

[FULL_MESH]

x -.- Accept Mesh Parameters

At this point, we are ready to let ANA begin building the mesh, so enter ' $x$ ' to proceed. See the ANA manual for other possible options.

Enter Command: $\mathbf{x}$

NON_PAD GRID DIMENSIONS CALCULATED TO BE

$$
\begin{aligned}
& I=40 \\
& J=40 \\
& K=44
\end{aligned}
$$

EXTENT OF BOUNDING RPP IN MILLIMETERS

$$
\begin{array}{lll} 
& \text { MIN MAX } \\
\mathrm{X} & -1000.00 & 1000.00 \\
\mathrm{Y} & -1000.00 & 1000.00 \\
\mathrm{Z} & -1150.00 & 1000.00
\end{array}
$$

Mesh Parameters for Group [1]

Grid Alignment

Number of Boundary Pad Cells

Set Cell Size

Set Ray Distribution

Output Fields

Mesh Format

Mesh File Name

Max Rays Per Cell

Cast Rays on Solid Centers
$[0.00][0.00][0.00]$

[8]

[50.00]

[RANDOM]

[ELECTRIC]

[CELL_ASC]

[sphere]

[4][0][4]

[OFF

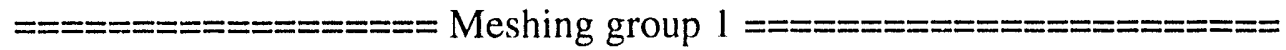

cylinder.r carved_sphere.r

Max Rays $=[4][0][4]$

[0] [1] [2] [3] [4] [5] [6] [7] [8] [9] [10] [11] [12] [13] [14] [15] [16]

[17] [18] [19] [20] [21] [22] [23] [24] [25] [26] [27] [28] [29] [30] [31]

[32] [33] [34] [35] [36] [37] [38] [39]

Group 1 Meshed in 56.800000 CPU seconds

When Ana is finished, three new files should exist: ana_ERR,hollow_s_LOG and sphere_a_cla. The file ana_ERR will contain any error messages written during the meshing procedure, hollow_s_LOS will contain a record of the meshing session, and 
sphere_a_cla will contain the mesh itself: Standard procedure is to rename the mesh file, replacing the _a_cla string with a .grd extension. This practice will ensure that a grid created at one cell size will not be inadvertantly overwritten if the model is re-meshed at a different cell size or for some other reason. Also, it is good practice to include some mention of the cell size as part of the file name, in the event that more than one mesh is created. In the case of this example, the file will be renarned from sphere_a_cla to sphere_50.grd.

mv sphere_a_cla sphere_50.grd 


\section{Chapter 4}

\section{EXAMINING THE FINITE DIFFERENCE MESH}

At this point, a finite-dilference mesh exists which could be used as input to TSAR. However, we don't know if the mesh really is a good approximation to the solid model, or whether there are problems with the mesh. This can only be determined by examining the mesh itself, by using the program IMAGE. Invoke IMAGE by typing:

\section{image sphere_50.grd}

The program will open a window and read in the mesh. Once the mesh is completely read in, the user can manipulate what is displayed by using the various buttons arrayed on the screen. As described in the IMAGE manual, the full mesh can be displayed, or the user may display only a portion by changing the bounding box or selecting a single plane for display.

To display the full mesh, click on 'Fields' with the left mouse button (all IMAGE commands use the left mouse button). This will cause the full mesh to be displayed. At $50 \mathrm{~mm}$ resolution, the example model is a particularly dense mesh, and trying to work with the full model will be extremely slow. Instead, click on the 'Bbox only' button to turn off the display, then click on 'Yrot', move the pointer over the slide bar along the right hand side of the display window, hold down the left mouse button and move the mouse up and down until the y rotation is approximately 90 degrees.

Now try looking at the mesh a single plane at a time, by clicking on the ' $i$ slice' button, then the 'Fields' button. The display should show a single plane through the middle of the mesh, and the dielectric cylinder should be visible. Try out some of the other buttons, referring to the IMAGE manual as necessary. When you are finished, click on the 'exit' button along the top to leave IMAGE.

The idea is to look for gaps where there should be no aperture, materials where there should be none, dangling edges, lack of symmetry, etc. Look carefully at the apertures and at the cylinder piercing the sphere. If the result doesn't look correct, it may be necessary to return to ANA and try re-gridding with some different parameters. You may need to try adjusting mesh alignment, or changing the cell size slightly, reordering material numbers, (higher numbered materials take precedence), or meshing some parts in different groups. This is an extremely important step in the modeling process, and several iterations may be necessary before a satisfactory mesh is obtained.

An important point is the material precedence scheme used by ANA. Look carefully at the cylinder piercing the sphere. Notice how the cylinder appears to shrink in diameter where it enters the sphere. This is because where there is a material conflict, ANA will choose the higher-numbered material. Since the cylinder in this case is number three and the sphere is number eight, the sphere will be given precedence, which results in a slightly smaller cylinder. A better choice might have been to give the cylinder a higher material number. This would require returning to MGED and re-doing the mater command for the sphere and the cylinder, then re-running ANA. For the purpose of this tutorial, we will just keep the current model.

Because the mesh is just an approximation to the solid model, it will be impossible to obtain a perfect mesh. Only the user can decide if a particular mesh is a good electromagnetic representation of the object to be simulated, and this must be based on a knowledge of EM, experience in running TSAR, and on a certain amount of intuition. 
Don't overlook the importance of this step. Usually ANA will provide a reasonably good mesh, but if any errors or anomalies do exist, the answers that TSAR provides will not accurately reflect what happens to the real object which you are trying to simulate. 


\section{Chapter 5}

\section{SETTING TSAR PARAME'TERS}

Once an cceptable finite-difference mesh has been obtained, the preliminary work has been completed, and it is now time to begin to work with TSAR itself. A program called SETUP_TSAR, (formerly called SHELL), exists to help set up both the parameters necessary to compile TSAR and the input file used to actually run TSAR. To run the program, simply type:

\section{setup_tsar} TSAR.

The following is the session used to set up the parameter files necessary to compile

SETUP_TSAR - an interactive utility for setting up TSAR input files

(C) Copyright 1988, 1991, 1992.

The Regents of the Unversity of California. All rights reserved

$$
\text { SETUP_TSAR }
$$

1. Enter/Modify basic compile-time parameters.

2. Enter/Modify far-field compile-time parameters.

3. Enter/Modify thin slot compile-time parameters.

4. Enter/Modify run-time namelist file.

5. Exit.

(* indicates that an item of this type exists.)

Please select an option $>>$

1

Selecting 1 calls up a menu which will allow arrays within TSAR to be specified for compilation.
\# Description
Current value

1. Number of cells in the I direction

20

2. Number of cells in the J direction

3. Number of cells in the $\mathrm{K}$ direction

4. J planes in core memory

5. Maximum \# of point field sensors

6. Maximum \# of internal radiators

10

7. Maximum \# of slice sensors

4

8. Maximum length of user-supplied pulses

9. Maximum \# of user-supplied pulses

10. Maximum \# of volumee sensors

11. Number of bits for each material index

12. Name of NAMELIST user input file

13. Exit

tsarin

Numbers 1, 2 and 3 need to be changed to match the values found in the header of the mesh file. In this case, $\mathrm{I}=56, \mathrm{~J}=56$ and $\mathrm{K}=60$. 
What item would you like to change? >>

1

$\begin{array}{ll}\text { Item to change } & : \text { Maxl } \\ \text { Type } & : \text { Integer } \\ \text { Lower Limit } & : 0 \\ \text { Current Value } & : 20\end{array}$

New value $>>$

\section{6}

\# Description Current value

*1. Number of cells in the 1 direction 56

2. Number of cells in the J direction 20

3 . Number of cells in the K direction 20

4. J planes in core memory 20

5. Maximum \# of point field sensors 10

6. Maximum \# of internal radiators 10

7. Maximum \# of slice sensors 4

8. Maximum length of user-supplied pulses 200

9. Maximum \# of user-supplied pulses 4

10. Maximum \# of volumee sensors 1

11. Number of bits for each material index 3

12. Name of NAMELIST user input file tsarin

13. Exit

What item would you like to change? $>$

2

Item to change : MaxJ

Type : Integer

Lower Limit $\quad: 0$

Current Value : 20

New value $>>$

56

\# Description Current value

*1. Number of cells in the I direction 56

*2. Number of cells in the J direction 56

3 . Number of cells in the K direction 20

4. J planes in core memory 20

5. Maximum \# of point field sensors 10

6. Maximum \# of internal radiators 10

7. Maximum \# of slice sensors 4

8. Maximum length of user-supplied pulses 200

9. Maximum \# of user-supplied pulses 4 
10. Maximum \# of volume sensors

11. Number of bits for each malerial index

1

12. Name of NAMELIST user input file

13. Exit

What item would you like to change? >>

3

$\begin{array}{ll}\text { Item to change } & \text { : MaxK } \\ \text { Type } & \text { Integer } \\ \text { Lower Limit } & : 0 \\ \text { Current Value } & : 20\end{array}$

New value $>>$

60

\# Description

Current value

* 1. Number of cells in the I direction

* 2. Number of cells in the J direction

* 3. Number of cells in the K direction

4. J planes in core memory

5. Maximum \# of point field sensors

10

6. Maximum \# of internal radiators

10

7. Maximum \# of slice sensors

4

8. Maximum length of user-supplied pulses

9. Maximum \# of user-supplied pulses

10. Maximum \# of volumee sensors

11. Number of bits for each material index

12. Name of NAMELIST user input file

13. Exit

4

1

3

tsarin

All the $\mathrm{J}$ planes should fit into memory, so set number 4 equal to the number of $\mathrm{J}$ planes.

What item would you like to change? >>

4

$\begin{array}{ll}\text { Item to change } & \text { : JBufSize } \\ \text { Type } & : \text { Integer } \\ \text { Lower Limit } & : 0 \\ \text { Current Villue } & : 20\end{array}$

New value $>>$

56

\# Description Current value

* 1. Number of cells in the I direction

* 2. Number of cells in the J direction

* 3. Number of cells in the K direction 
*4. J planes in core memory

5. Maximum \# of point field sensors

6. Maximum\# of internal radiators

7. Maximum \# of slice sensors 4

8. Maximum length of user-supplied pulses 2()()

9. Maximum \# of user-supplied pulses 4

10. Maximum \# of volume sensors 1

11. Number of bits for each material index 3

12. Name of NAMELIST user input file tsarin

13. Exil

For this example problem, we may keep the default values for the rest of the parameters. See the TSAR manual for an explanation of these parameters.

To exit this menu, choose number 1.3 .

What item would you like to change? >>

13

\section{SETUP_TSAR}

*1. Enter/Modify basic compile-time parameters.

2. Enter/Modify far-field compile-time parameters.

3. Enter/Modify thin slot compile-time parameters.

4. Enter/Modify run-time namelist file.

5. Exit.

(* indicates that an item of this type exists.)

Please select an option $>>$

At this point, we have created the file params.inc, which contains some of the parameters necessary to compile the tsar code for this size problem. The next step is to set the farfield projection parameters.

In this problem, we don't intend to perform any far-field calculations, so the default values will be fine. However, on some machines, in order to get the file projprms.inc written to the current directory, it is necessary to enter at least one value, even if it is the same as the default. (An alternative would be to copy projprms.ctp from the tsar source file, edit it as necessary and rename it projprms.inc.) Choose item 2 from the menu to select the far-field parameters, then enter one for the maximum number of frequencydomain far-field points per pattern.

Please select an option $>$

\section{2}

\# Description

Current value

1. Max \# of freq-domain far-field patterns

2. Max \# of freq-domain far-field points per pattern

3. Max \# of time-domain far-field observation points

4. Max \# of time-domain far-field timesteps per point

5. Max \# of time-domain near-field observation points 
6. Max \# of space points in neilr-field simpling surfice

7. Max \# of time-domain near-fied timesteps per point

8. lixil

What item would you like to change? >>

2

llem to change : MaxFFPts

Type : Integer

Lower limil : 0

Current Value : 1

New vallue $>$

1

Selecting a menu item, even if the entry is the same as the default, is sufficient to caltuse the include file to be created.
\# Description
Current value

1. Max\# of freq-domain far-field patterns

* 2. Max \# of freq-domain far-field points per pattern

3. Max \# of time-domain far-field observation points

4. Max \# of time-domain far-field timesteps per point

5. Max \# of time-domain near-field observation points

6. Max \# of space points in near-field sampling surface

7. Max \# of time-domain near-field timesteps per point

8. Exit

What item would you like to ch'inge? >>

8

\section{SETUP_TSAR}

* 1. Enter/Modify basic compile-time parameters.

* 2. Enter/Modify far-field compile-time parameters.

3. Enter/Modify thin slot compile-time parameters.

4. Enter/Modify run-time namelist file.

5. Exit.

(* indicates that an item of this type exists.)

Please select an option $>>$

Having completed item 2 on the menu, we have now dimensioned the problem and have created two files, params.inc and projprms.inc. We next need to create the file slotprms.inc, but we don't need to modify the parameters since there are no thin slots (less 
than a cell dimension) in this example problem. Entering, touching one value, then exiting the menu will create the necessary lile.

3

\# Description Current value

1. Max \# of thin slots allowed

2. Max \# of cells with thin slots

3. Max \# of basis functions per slot

4. Max \# of time steps saved in HTSA

5. Max \# of HTSA probes

6. Exit

What item would you like to change? >>

I

$\begin{array}{ll}\text { Item to change } & \text { :MaxSlots } \\ \text { Type } & \text { :Integer } \\ \text { Lower Limit } & : 1 \\ \text { Current Value } & : 1\end{array}$

New value $>>$

I

\# Description Current value

* 1. Max \# of thin slots allowed

2. Max \# of cells with thin slots

3. Max \# of basis functions per slot

4. Max \# of time steps saved in HTSA

5. Max \# of HTSA probes

6. Exit

What item would you like to change? $>$

6

\section{SETUP_TSAR}

* 1. Enter/Modify basic compile -time parameters.

* 2. Enter/Modify far-field compile-time parameters.

* 3. Enter/Modify thin slot compile-time parameters.

4. Enter/Modify run-time namelist file.

5. Exit.

(* indicates that an item of this type exists.)

Please select an option $>$

Next, before leaving setup_tsar, we need to create an input file for a particular tsar run, which will include such things as which boundary conditions to use, how many time steps 
to run, etc. To create the input file, usually called tsarin, select mumber four from the menllu.

Please select an option >

4

\section{SIMULATION PARAMETERS}

1. Generial problem set-up

2. Materials

3. Incident wave shape

4. Point radiattion sources

5. External plane wave sources

6. Point sensors

7. Input verificiation

8. Near-field sampling

9. Far-field projections

10. Thin Slots

11. Slice sensors

12. Volume sensors

13. Finished

Select an option >>

\section{1}

\# Description Current value

1. Comment title of problem

TSAR Finite Difference Software

2. Size of single grid cell

3. Total number of time steps to execute

$4 \quad$ Use explicit virtual memory?

5. Name of object grid file

6. Use real, not grid coordinates?

7. I location of real origin

8. J location of real origin

9. K location of real origin

10. Boundary condition of XMin face

11. Boundary condition of XMax face

12. Boundary condition of YMin face

13. Boundary condition of YMax face

14. Boundary condition of ZMin face

15. Boundary condition of ZMax face

16. Exit

What item would you like to change'? >>

1

Item to change : Title

Type :String 
Current Value : TSAR Finite Difference Soltware

Enter new value $>>$

Hollow_sphere,dx=5() $\mathrm{mm}, 9 / 1 / 93$

\# Description Current value

*1. Comment title of problem Hollow_sphere, $\mathrm{d} x=50 \mathrm{~mm}, 9 / 1 / 93$

2. Size of single grid cell

3. Total number of time steps to execute 16

4. Use explicit virtual memory? NO

5. Name of object grid file grid

6. Use real, not grid coordinates? NO

7. Ilocation of real origin $0.000 \mathrm{E}+00$

8. J location of real origin $0.000 \mathrm{E}+00$

9. $\mathrm{K}$ locition of real origin $\quad 0.000 \mathrm{E}+00$

10. Boundary condition of XMin face None

11. Boundary condition of XMax face None

12. Boundary condition of YMin face None

13. Boundary condition of YMax face None

14. Boundary condition of ZMin face None

15. Boundary condition of ZMax face None

16. Exit

What item would you like to change? >>

1

Select number two to set the proper cell size.

Item to change $\quad: \mathrm{Dx}$

Type : Real

Lower Limit $\quad: 0$.

Current Value $\quad: 1.000 \mathrm{E}-02$

New value $>>$

\section{0e-2}

\# Description Current value

\begin{tabular}{|c|c|c|}
\hline$* 1$. & Comment title of problem & $=50 \mathrm{~mm}, 9 / 1 / 93$ \\
\hline *2. & Size of single grid cell & $5.000 \mathrm{E}-02$ \\
\hline 3. & Total number of ime steps to execute & 16 \\
\hline 4. & Use explicit virtual memory? & $\mathrm{NO}$ \\
\hline 5. & Name of object grid file & grid \\
\hline 6. & Use real, not grid coordinates? & NO \\
\hline 7. & I location of real origin & $0.000 \mathrm{E}+00$ \\
\hline 8. & $\mathrm{~J}$ location of real origin & $0.000 \mathrm{E}+00$ \\
\hline 9. & $\mathrm{~K}$ location of real origin & $0.000 \mathrm{E}+00$ \\
\hline 10. & Boundary condition of XMin face & None \\
\hline 11. & Boundary condition of XMax face & None \\
\hline 12. & Boundary condition of YMin face & None \\
\hline
\end{tabular}


13. Boundary condition of YMax facce

None

14. Boundary condition of ZMin liace

None

15. Boundary condition of ZMax lace

Nonc

16. Exit

What item would you like to change? >>

Set the number of time steps for the problem to run by selecting number three.

We will run the problem for 128 time steps.

What item would you like to change? >>

3

ltem to change : NSteps

Type : Integer

Lower limit : : ()

Current Value : 16

New vallue $>>$

128

\# Description Current value

\begin{tabular}{|c|c|c|}
\hline$* 1$. & Comment title of problem & Hollow_sphere, $\mathrm{dx}=50 \mathrm{~mm}, 9 / 1 / 93$ \\
\hline$* 2$. & Size of single grid cell & $5.000 \mathrm{E}-() 2$ \\
\hline *3. & Total number of time steps to execute & 128 \\
\hline 4. & Use explicit virtual memory? & $\mathrm{NO}$ \\
\hline 5 & Name of object grid file & grid \\
\hline 6. & Use real, not grid coordinates? & NO \\
\hline 7. & I location of real origin & $.000 \mathrm{E}+00$ \\
\hline 8. & J location of real origin & $0.000 \mathrm{E}+00$ \\
\hline 9 & $\mathrm{~K}$ location of real origin & $0.000 \mathrm{E}+00$ \\
\hline 10. & Boundary condition of XMin face & None \\
\hline 11. & Boundary condition of XMax face & None \\
\hline 12. & Boundary condition of YMin face & None \\
\hline$i 3$. & Boundary condition of YMax face & None \\
\hline 14. & Boundary condition of ZMin face & None \\
\hline $\begin{array}{l}15 . \\
16 .\end{array}$ & $\begin{array}{l}\text { Boundary condition of ZMax face } \\
\text { Exit }\end{array}$ & None \\
\hline
\end{tabular}

What item would you like to change? $>>$

5

$\begin{array}{ll}\text { Item to change } & : \text { GridFile } \\ \text { Type } & : \text { String } \\ \text { Current Value } & : \text { grid }\end{array}$

Enter new value $>>$

sphere 50.grd 
*1. Comment tille if problem

*2. Size of single erid eell

*3. Tolal mumber of time steps to execute

Hollow sphere, $(\mathrm{dx}=5() \mathrm{mm}, 9 / \mathrm{l} / 93$

4. Use explicil virtual memory?

*5. Name of object grid file

6. Ise real, not grid coordinates?

7. I locallom of real origin

8. J localtion of real origin

). K locittion of real origin

10. Boundary condition of XMin face

11. Boundary condition of XMax face

12. Boundary condition of YMin face

13. Boundary condition of YMax face

14. Boundary condition of ZMin face

15. Boundary condition of ZMax lace

10. Exit

$5 .(0)(0) \mathrm{E}-() 2$

128

NO

sphere 50.grd

NO

$(0.00(0 \mathrm{E}+() 0$

(). (0)(OE+ $+(0)$

$0.000 \mathrm{E}+00$

None

None

None

None

None

None

What item would you like to change? >>

10

Item to change

Type

Possible values

\author{
: BCXMin \\ : String \\ NONE \\ MAGNETIC PERFECT CONDUCTOR \\ ELECTRIC PERFECT CONDUC'TOR \\ 2ND ORDER MUR RADIATION \\ IST ORDER MUR RADIATION
}

Current Value : None

Enter new value $>>$

2nd

\# Description

Current value

*1. Comment title of problem

*2. Size of single grid cell

*3. Total number of time steps to execute

Hollow_sphere, $\mathrm{dx}=50 \mathrm{~mm}, 9 / 1 / 93$

4. Use explicit virtual memory?

*5. Name of object grid lile

6. Use real, not grid coordinates'?

7. I location of real origin

8. J location of real origin

9. K locition of real origin

* 10. Boundary condition of XMin face

11. Boundary condition of XMax face

12. Boundary condition of YMin face

13. Boundary condition of YMax face

5.000E-02

128

NO

sphere50.grd

NO

$0.000 \mathrm{E}+00$

$0.000 \mathrm{E}+00$

$0.000 \mathrm{E}+00$

2ND ORDER MUR RADIATION

None

None

None 
14. Boundary condition of ZMin face

None

15. Boundary condition of ZMax face

None

16. Exit

What item would you like to change? >>

11

Item to change : BCXMax

Type

: String

Possible values : NONE

MAGNETIC PERFECT CONDUCTOR

ELECTRIC PERFECT CONDUCTOR

2ND ORDER MUR RADIATION

IST ORDER MUR RADIATION

Current Value : None

Enter new value $>>$

2nd

\# Description Current value

*1. Comment title of problem

*2. Size of single grid cell

*3. Total number of time steps to execute

Hollow_sphere, $\mathrm{dx}=50 \mathrm{~mm}, 9 / 1 / 93$

$5.000 \mathrm{E}-02$

4. Use explicit virtual memory?

*5. Name of object grid file

6. Use real, not grid coordinates?

7. I location of real origin

8. J location of real origin

9. K location of real origin

128

NO

sphere_50.grd

NO

$0.000 \mathrm{E}+00$

$0.000 \mathrm{E}+00$

$0.000 \mathrm{E}+00$

* 10. Boundary condition of XMin face

*11. Boundary condition of XMax face

12. Boundary condition of YMin face

13. Boundary condition of YMax face

14. Boundary condition of ZMin face

15. Boundary condition of ZMax face

16. Exit

2ND ORDER MUR RADIATION

2ND ORDER MUR RADIATION

None

None

None

None

What item would you like to change? >>

Continue through the boundary conditions, setting each one to 2 nd order Mur, until all six faces have beer, set, then enter 16 to leave this menu.

\# Description Current value

*1. Comment title of problem

*2. Size of single grid cell

*3. Total number of time steps to execute

4. Use explicit virtual memory?
Hollow_sphere, $\mathrm{dx}=50 \mathrm{~mm}, 9 / 1 / 93$

$5.000 \mathrm{E}-02$

128

NO 
*5. Name of object grid file

6. Use real, not grid coordinates?

7. I location of real origin

8. J location of real origin

9. K location of real origin

* 10. Boundary condition of XMin face

*11. Boundary condition of XMax face

*12. Boundary condition of YMin face

* 13. Boundary condition of YMax face

*14. Boundary condition of ZMin face

* 15. Boundary condition of ZMax face 16. Exit sphere 50.grd

NO

(). $(000 \mathrm{E}+(0)$

$0.000 \mathrm{E}+00$

$0.000 \mathrm{E}+00$

2ND ORDER MUR RADIATION 2ND ORDER MUR RADIATION 2ND ORDER MUR RADIATION 2ND ORDER MUR RADIATION 2ND ORDER MUR RADIATION 2ND ORDER MUR RADIATION

What item would you like to change? >>

16

\section{SIMULATION PARAMETERS}

*1. General problem set-up

2. Materials

3. Incident wave shape

4. Point radiation sources

5. External plane wave sources

6. Point sensors

7. Input verification

8. Near-field sampling

9. Far-field projections

10. Thin Slots

11. Slice sensors

12. Volume sensors

13. Finished

Select an option $>>$

\section{2}

Next, the material constituitive parameters need to be set. Enter 2 to select the materials menu.

\# Description Current value

1. Relative material permittivity, epsilon

(Array)

2. Relative material permeability, mu

(Array)

(Array)

3. Electrical conductivity

4. Magnetic conductivity

5. Trim calcs outside closed shell?

(Array)

6. Exit

NO

What item would you like to change? >>

\section{1}

Start by setting any relative dielectric constants different than free space. 
Item to change : EpsR

Type : Reall Array

Lower Limit : 1.

Current Value ( 1): 1.00

Current Value (2): 1.00

Current Value ( 3): 1.00

Current Value (4): 1.00

Current Value ( 5): 1.00

Item to change, new value $(0,0$ to end $)>>$

To make the cylinder a dielectric, choose material 3 and enter a relative dielectric constant. Enter the material and constant as a pair separated by a comma.

\section{$3,1.7$}

$\begin{array}{ll}\text { Item to change } & \text { : EpsR } \\ \text { Type } & \text { : Real Array } \\ \text { Lower Limit } & : 1 . \\ \text { Current Value ( 1) : } & 1.00 \\ \text { Current Value (2): } & 1.00 \\ \text { Current Value (3): } & 1.70 \\ \text { Current Value (4): } & 1.00 \\ \text { Current Value (5): } & 1.00\end{array}$

Item to change, new value $(0,0$ to end $)>>$

To exit this menu, enter 0,0 .

$\mathbf{0 , 0}$

\# Description Current value

$\begin{array}{rll}\text { *1. Relative material permittivity, epsilon } & \text { (Array) } \\ \text { 2. Relative material permeability, mu } & \text { (Array) } \\ \text { 3. Electrical conductivity } & \text { (Array) } \\ \text { 4. Magnetic conductivity } & \text { Array) } \\ \text { 5. Trim calcs outside closed shell? } & \text { NO }\end{array}$

6. Exit

What item would you like to change? >>

Make the sphere metallic by choosing an electrical conductivity value.

3

Item to change $\quad$ : ConductE

Type : Real Array

Lower Limit : $\quad 0$.

Current Value ( 1): $0.000 \mathrm{E}+00$

Current Value ( 2): $0.000 \mathrm{E}+00$

Current Value ( 3): $0.000 \mathrm{E}+00$

Current Value (4): $0.000 \mathrm{E}+00$

Current Value ( 5) : $0.000 \mathrm{E}+00$ 
Item to change, new value $(0,0$ to end $)>>$

The sphere is material 8 . Even though 8 is not displayed, it can still be entered as a material value. The menu will change to display 5 values centered at the chosen material number.

\section{8,3.0e}

Item to change : ConductE

Type : Real Array

Lower Limit : 0 .

Current Value (4): $0.000 \mathrm{E}+00$

Current Value ( 5): $0.000 \mathrm{E}+00$

Current Value (6) : $0.000 \mathrm{E}+00$

Current Value ( 7) : $0.000 \mathrm{E}+00$

Current Value ( 8) : $3.000 \mathrm{E}+07$

Item to change, new value $(0,0$ to end $)>>$

Exit this menu by entering 0,0 .

\section{$\mathbf{0 , 0}$}

\# Description Current value

$\begin{array}{rll}* 1 . & \text { Relative material permittivity, epsilon } & \text { (Array) } \\ \text { 2. } & \text { Relative material permeability, mu } & \text { (Array) } \\ \text { 3. } & \text { Electrical conductivity } & \text { (Array) } \\ \text { 4. Magnetic conductivity } & \text { (Array) } \\ \text { 5. Trim calcs outside closed shell? } & \text { NO }\end{array}$

6. Exit

What item would you like to change? >>

Having defined values for all the regions in our model, we can exit.

6

\section{SIMULATION PARAMETERS}

*1. General problem set-up

*2. Materials

3. Incident wave shape

4. Point radiation sources

5. External plane wave sources

6. Point sensors

7. Input verification

8. Near-field sampling

9. Far-field projections

10. Thin Slots

11. Slice sensors

12. Volume sensors 


\section{3. linished}

Select an option >>

\section{3}

The time history of the incident field now needs to be specified. Choose three from the menll.
\# Description
Current vilue

1. Shape of incident pulse

2

2. Trequency of sinusoidal waves

3. Time delay of Gaussian peak

$1.00(0 \mathrm{E}+(09$

4. Full width half-max of Ciaussian

40.0

25.0

16.0

10.0

6. Fall time of exponential pulses

1.00

7. Peak amplitude of incident fields

YES

9. File name of user-supplied pulses

(Array)

10. Exit

What item would you like to change'? >>

These menu items define the type of pulse and its particular shape. We'll keep the default parameters for this example. See the TSAR manual for an explanation.

10

\section{SIMULATION PARAMETERS}

*1. General problem set-up

*2. Materials

*3. Incident wave shape

4. Point radiation sources

5. External plane wave sources

6. Point sensors

7. Input verification

8. Near-field sampling

9. Far-field projections

10. Thin Slots

11. Slice sensors

12. Volume sensors

13. Finished

Select an option $>>$

We want to excite our model externally, so we don't want to set any radiation sources. Our next choice should be number five.

\section{5}


1. Apply external plane wave?

2. Run 'TSAR in scatlered ficld mode"?

3. Elevattion angle of incident plane wave

4. Azimuthal angle of incident plane wave

5. Polarization of incident plane wave

6. Material index of surrounding medium

7. User pulse shape for exlermal waves

()

8. XMin boundary of total lied zone

9. XMax boundary of total field zone

10. YMin boundary of total lield zone

11. Y'Max boundary of total field zone

12. ZMin boundary of total field zone

13. ZMax houndary of total field zone

14. Exit

What item would you like lo change? >>

\section{1}

Changing one will excite the problem with a plane wave. Numbers three through five dictate the direction and polarization of the wave. Match the numbers below lo set up a plane wave propagating in the $x$-direction, polarized in the $y$-direction.

\# Description

*1. Apply external plane wave?

2. Run TSAR in scattered field mode?

3. Elevation angle of incident plane wave

*4. Azimuthal angle of incident plane wave

*5. Polarization of incident plane wave

6. Material index of surrounding medium

7. User pulse shape for external waves

8. XMin boundary of total field zone

9. XMax boundary of total field zone

10. YMin boundary of total field zone

11. YMax boundary of total field zone

12. ZMin boundary of total field zone

13. ZMax boundary of total field zone

14. Exit

Current value

What item would you like to change? >>

14

\section{SIMULATION PARAMETERS}

*1. General problem set-up

*2. Materials

*3. Incident wave shape

4. Point radiation sources

*5. External plane wave sources

6. Point sensors 
7. Input verification

8. Near-ficld simpling

9. Far-licld projections

10. Thin Slots

11. Slice sensors

12. Volume sensors

1.3. Finished

Sclect ill option >>

We now have the simulation itedf defined. We next need to choose the output from TSAR. Let's select two point sensors from menu number 6 and a sliee sensor from menu II.

6

\# Description Current value

1. X location of point sensors

2. Y location of point sensors

(Array)

(Arruy)

3. Z location of point sensors

(Array)

4. $X$ component of point direction

(Array)

5. $Y$ component of point direction

(Array)

6. Z Component of point direction

(Array)

7. Type of point sensors

(Array)

8 Turn-on time of point sensors

(Array)

9. Turn-off time of point sensors

(Array)

10. Prefix name of point sensor data files

II Print point data in binary (vs ASCII)?

point

12. Exit

NO

What item would you like to change? >>

First set the location of the sensors, in grid $(\mathrm{I}, \mathrm{J}, \mathrm{K})$ co-ordinates.

1

Item to change : PntX

Type : Real Array

Current Value (1): (Not Assigned)

Current Value (2) : (Not Assigned)

Current Value (3): (Not Assigned)

Current Value (4): (Not Assigned)

Current Value ( 5): (Not Assigned)

llem to change, new value (1),(0) to end) $>>$

\section{1,28}

Item to change : PntX

Type : Real Array

Current Value (1): 28.0

Current Value ( 2) : (Not Assigned)

Current Value ( 3) : (Not Assigned) 
Current Value (4): (Nol Assigned)

Current Valle ( 5): (Nol Assigned)

Item to change, new value (1),() to end) $>>$

2,20

llem to change : PntX

Type : Real Array

Current Vallue (1): 28.0

Current Villue (2): 20.0

Current Value ( 3): (Not Assigned)

Current Value (4): (Not Assigned)

Current Value ( 5): (Not Assigned)

Item to change, new value $(0,0)$ to end $)>>$

(),()

\# Description

Current value

$\begin{array}{rll}\text { *1. X locution of point sensors } & \text { (Array) } \\ \text { 2. Y location of point sensors } & \text { (Array) } \\ \text { 3. Z location of point sensors } & \text { (Array) } \\ \text { 4. X component of point direction } & \text { (Array) } \\ \text { 5. Y component of point direction } & \text { (Array) } \\ \text { 6. Z component of point direction } & \text { (Array) } \\ \text { 7. Type of point sensors } & \text { (Array) } \\ \text { 8. Turn-on time of point sensors } & \text { (Array) } \\ \text { 9. Turn-off time of point sensors } & \text { (Array) } \\ \text { 10. Prefix name of point sensor data files } & \text { point } \\ \text { 11. Print point data in binary (vs ASCII)? } & \text { NO } \\ \text { 12. Exit } & \end{array}$

What item would you like to change? >>

Proceed through the menu, setting $y$ and $z$ locations for probes 1 and 2. Use IMAGE to assure that the probes are located in the appropriate locations. Also specify a probe direction for probes 1 and 2 by setting a value for each of the component directions. The default setting is $z=1.0$. The total length must not exceed 1 , so if another component is selected, reduce the z component appropriately. In this case, the incident field is polarized in the y direction, so a good choice might be to set the $y$-components to I and reduce the $z$-components to 0 .

Finally, choose a probe type, such as EFIELD. Refer to the TSAR manual for the choices.

\# Description Current value

*1. X location of point sensors

(Array)

*2. Y location of point sensors

(Array)

*3. Z Z location of point sensors

(Array)

4. X component of point direction

(Array) 
*5. Y compunent of point direction

(Arrily)

*h. $\%$ component of point direction

(Arrily)

*7. Type of point sensors

8. fiurn-en lime of point sensors

(Arrily)

(Array)

9. Turn-olt time of point sensors

(Arrily)

11. Prefix name of point sensor data fïles

11. Print point dalta in binary (vs ASCII) ?

point

12. Exil

N()

What item would you like to change!? >>

12

\section{SIMULATION PARAMETTERS}

* I. Cieneral problem set-up

*2. Millerials

*3. Incident wave shape

4. Point radiation sources

*5. External plane wave sources

*6. Point sensors

7. Input verification

8. Near-field sumpling

9. Fir-ficld projections

10. Thin Slots

11. Slice sensors

12. Volume sensors

13. Finished

Select an option $\quad>$

11

\# Description

Current vilue

1. Prefix name of slice output data files

slice

2. Type of slice sensor

3. Switch to turn slice sensors on and ofi

Array)

(Arrily)

4. Turn-on time of slice sensors

5. Turn-off time of slice sensors

6. Time step increment for slice sensors

(Array)

(Array)

(Array)

7. Lower I limit for slice sensors

(Array)

8. Upper I limit for slice sensors

(Arriiy)

9. I increment for slice sensors

10. Lower J limit for slice sensors

(Arrily)

11. Upper J limit for slice sensors

(Array)

12. J increment for slice sensors

(Arrily)

13. Lower $\mathrm{K}$ limit for slice sensors

(Arrily)

(Arrily)

14. Upper K limit for slice sensors

(Arrily)

15. K increment for slice sensors

(Array)

16. Exit 
Whall ilem would you like en change? >>

2

lem lo change : (Slckind

Type : String Array

Possible villess

$: \mathrm{I:X}$

I:Y

I:\%

IIX

IIY

HY

Courent Value (1):

Current Value (2):

Current Value (3):

current Vulue (4):

lem to change (0) fo end) >>

1

Enter new vilue $>$

EY

llem to change : CSlckind

Type : String Arrily

Possible values $\quad$ : EX

$E Y$
$E Z$
$H X$
$H Y$
$H Z$

Current Value ( 1): EY

Current Value (2):

Current Value ( 3):

Current Value (4):

Item to change $(0$ to end $)>>$

()

\# Description Current value

1. Prefix name of slice output data files

slice

*2. Type of slice sensor

3. Switch to turn slice sensors on and off

(Array)

4. Turn-on time of slice sensors

(Array)

5. Turn-off time of slice sensors

(Array)

6. Time step increment for slice sensors

(Array)

(Array)

7. Lower I limit for slice sensors

(Array) 
8. IPper I limit for slice semsers

9. I increment lor slice semsors

10. lower J limil for slice sellsors

11. Ipper J limit for slice semsors

12. J increment for slice sensors

13. Lower K limil for slice sensors

14. Upper K limit for slice sensors

15. Kincrement for slice sensors

16. Iixil
(Arrily)

(Arrily)

(Arrily)

(Arrity)

(Arrily)

(Arriy)

(Arrily)

(Arrily)

What item would you like to change? >>

Proceed through the items in this menu, giving a value to the first it element of each item. For at least one of the co-(ordinates, the upper and lower limits must be equal, so that a single plane is defined. (Volume sensors are available if desired).

\section{6}

\section{SIMULATION PARAMETERS}

*1. Cieneral problem sel-up

*2. Matterials

*3. Incident wave shape

4. Point radialion someces

*5. Extermal plane wave sources

*6. Point sensors

7. Input verificition

8. Near-field simmpling

9. Fir-lield projections

1(). Thin Slots

* II. Slice sensors

12. Volume sensors

13. Finished

\section{Select an option $>>$}

1.3

\section{SI:TUP TSAR}

* 1. Enter/Modify basic compile-time parameters.

* 2. Enter/Modily liar-field compile-time parameters.

* 3. Enter/Modily thin slot compile-time parameters.

* 4. Enter/Modify run-time namelist file.

5. lixit.

(* indicates that an item of this type exists.)

Please select an option >>

5

Call again!

STOP 
At this point. there are three include files, to be used for compiling 'TSAR, and one tsarin file, Io run a particular simulation. If any of these files need to be changed, either SLTUP TSAR or your fitvorite editor may be used at this stage.

The next step in the process is to compile an executable TSAR. If the installation instructions were followed. and your environment variables are properly set, it should only be necessiry 10 type:

\section{maketsur}

This command will lind the source code, compile and link TSAR, and leave an executable called tsar in the local directory. It will also leave a host of object files, *,o, in the local directory. Once a good executable has been built, these object filles can be deleted if desired.

It is a good idea to try TSAR for just a couple of time sleps the first time a new executuble is ready to run. This checks the settings in the tsarin file and will quickly let you know if something is not correctly set. Once it runs for a couple of time steps and creates the right output files, etc., one can edit the tsarin file for the desired number of lime steps. Since for any realistic job, TSAR can run for long periods of time, it is almost always submitted as a background job:

tsar $>\&$ tsar.log $\&$ 


\section{('haptero}

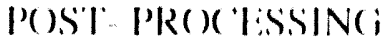

When TSAR finishes running, we should have three outpul files: point()1, point(1) and slice()I. The point files are in SIG external formall, that is. Ihey contain several lines of header information followed by just $Y$ values (implied $X$ values). These files can be read into Sig through the TSREAD command.

If a processing package other than SIC is lo be used. a simple filler can be written lo read the header, extract the intial time (TO) and the time between $Y$ valles (D'T), then rewrite the file with explicit $X$ values ( $X Y$ pairs). By only storing $Y$ values the data files are greally reduced in size. However, if your post-processor always reyuires $X Y$ pairs, it might be worthwhile to alter the output routines within TSAR.

The slice file output can be converted into a ras file with the program B2RAS, for displaty with XTRAS. To run B2RAS, type b2ras, followed by an input file and an output lile:

\section{b2ras slice()1 slice()1.rus}

Once again, this program can be run graphically, with mouse interaction, or in teletype mode, with a command line interface. The following is an example of TTY mode.

Select Mode for Option Selection ( graphics - lly ): tty

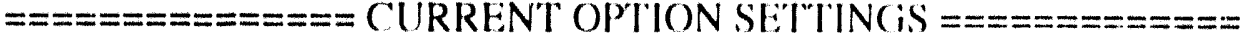

DATA OPTIONS

Merge geometry with processed datal

Fill artifacts in the mesh

Dalla is cell centered

Average pixels between data points

Process all frames in the input file

Do not bevel geometry edges

Ditla is not symettric about ()

Use linear data scaling

ORIENTATION OPTIONS

Do not rotate image

Do not flip image horizontally

Do not flip image vertically

( OI.OR TABLI:

Selected color table is |Multicolor I|

\section{B2RAS MAIN PARAMLITIR MINII ==============}

Make changes to B2RAS parameters by selecting the appropriate sub-menu.

c- change (olor lable

(1- change Dalla options

0 - change (Orientation options

$x$ - accept current options settings and proceed 
Enter desired option: c

COLOOR TABLE MENU

Current Color Table is [Multicolor I]

1 -.- Multicolor 1

2 -.. Multicolor 2

3 --- Multicolor 3

4 -.- Multicolor 4

5 -..- Multicolor 5

6 -..- Multicolor 6

7 -.- Multicolor 7

8 -.- Multicolor 8

() -.- Multicolor 9

1() ... Grayscale

Enter Color Table Number (1-10): 6

Color table 6 is a good choice for time domain values that are both positive and negative.

$===============$ CURRENT OPTION SETTINGS $==============$

DATA OPTIONS

Merge geometry $:$ ith processed data

Fill artifacts in the mesh

Data is cell centered

Average pixels between data points

Process all frames in the input file

Do not bevel geometry edges

Data is not symettric about 0

Use linear data scaling

ORIENTATION OPTIONS

Do not rotate image

Do not flip image horizontally

Do not flip image vertically

COLOR TABLE

Selected color table is [Multicolor 6]

$===============$ B2RAS MAIN PARAMETER MENU $===========$

Make changes to B2RAS parameters by seiecting the appropriate sub-menu.

c --- change Color table

d --- change Data options

o --- change Orientation options

$\mathrm{x}$-... accept current options settings and proceed

Enter desired option: $\mathbf{d}$

DATA OPTIONS MENU

d - Data

f - Fill Mesh

o- Data Orientation

a - Average between data values
[Data and Geometry]

[Fill]

[Cell Centered]

[Average] 
n - Process Frames

b - Bevel Mesh Edges

m - Data Is Symmetric About ()

$s$ - Data Scaling

$x$ - Accept Current Data Option Values

|All Frames|

$|\mathrm{Nr}|$

$[\mathrm{N}(1)]$

[Linear]

Enter character option: o

Data Orientation Can Be One of the Following:

c - Cell Centered

$\mathrm{x}$ - Along Ex

$y$ - Along Ey

z. - Along Ez.

Enter character: $\mathbf{y}$

Since we wrote Ey values, the natural choice is $y$.

\section{DATA OPTIONS MENU}
d - Data
f - Fill Mesh
o - Data Orientation
a - Average between data values
$n$ - Process Frames
b - Bevel Mesh Edges
m - Data Is Symmetric About 0
$s$ - Data Scaling
$\mathrm{x}$ - Accept Current Data Option Values

[Data and Geometry]

[Fill]

[Ey]

[Average]

[All Frames]

[No]

[No]

[Linear]

Enter character option: $\mathbf{m}$

Since these are time domain values, they vary about zero.

\section{DATA OPTIONS MENU}
d - Data
f - Fill Mesh
o - Data Orientation
a - Average between data values
n - Process Frames
b - Bevel Mesh Edges
m - Data Is Symmetric About ()
$s$ - Data Scaling
$\mathrm{x}$ - Accept Current Data Option Values

[Data and Geometry]

[Fill]

[Ey]

[Average]

[All Frames]

[No]

[Yes]

[Linear]

Enter character option: $s$

Enter number of cycles for log scaling: 2 
B2ras normalizes all values to the largest magnitude number found in the file. By choosing a 2-cycle log scale, the largest value in the output will be 1 and the smallest will be ()$,() 1$.

\section{DATA OPTIONS MENU}

$\begin{array}{ll}\text { d - Data } & \text { [Dala and Geometry] } \\ \text { f - Fill Mesh } & \text { [Fill] } \\ \text { o - Data Orientation } & \text { [Ey] } \\ \text { a - Average between data values } & \text { [Average] } \\ \text { n - Proces: Frames } & \text { [All Frames] } \\ \text { b - Bevel Mesh Edges } & \text { [No] } \\ \text { m - Data Is Symmetric About } 0 & \text { [Yes] } \\ \text { s - Data Scaling } & \text { [Log (2 cycles)] } \\ \text { x - Accept Current Data Option Values } & \end{array}$

Enter character option: $\mathbf{x}$

Accept the current data option settings and return to the main menu.

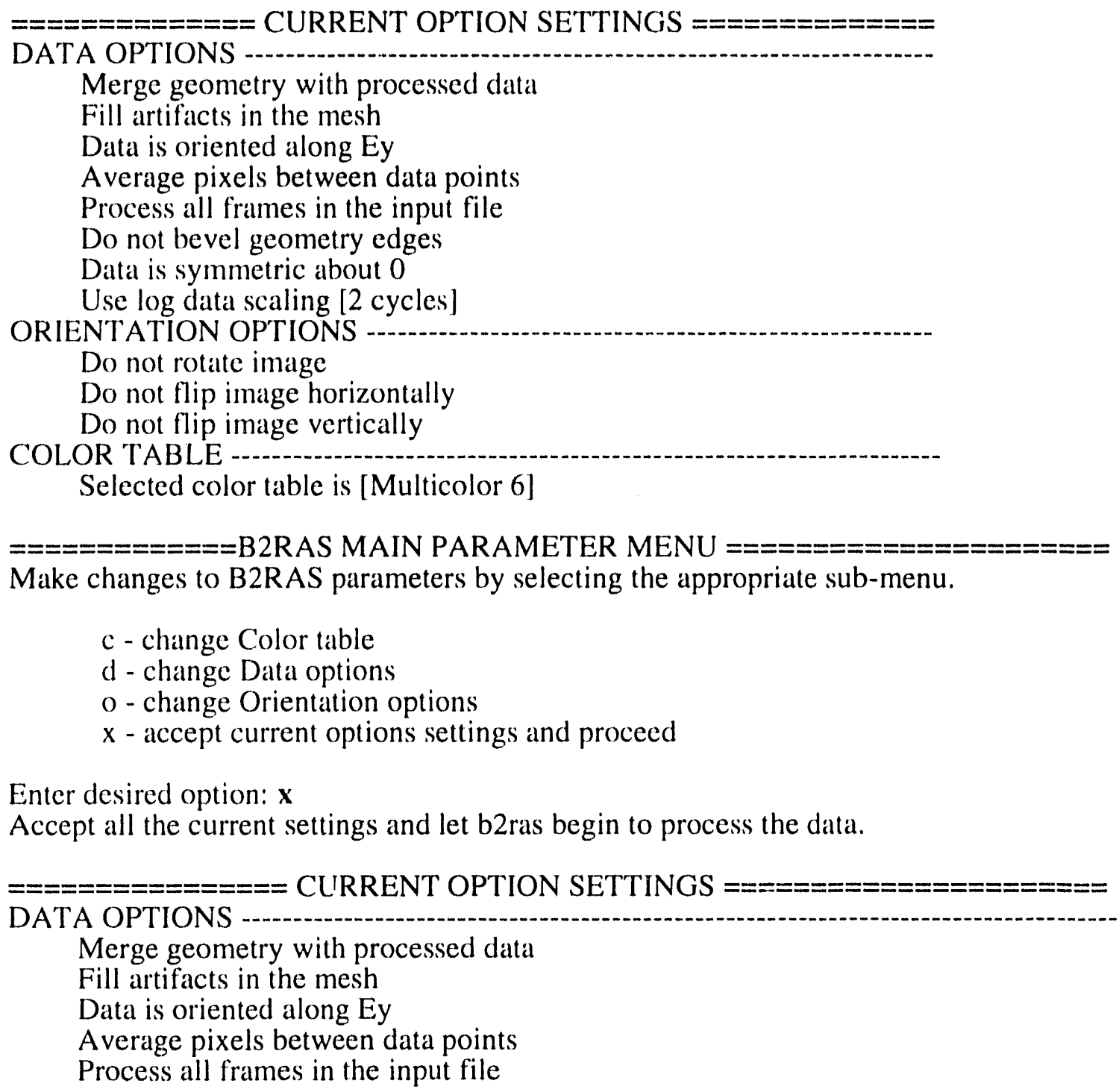


1) not bevel geomedry edges

Dalla is symmotric about ()

l se log dalta scilling | 2 cycles |

ORILENTATION OPTIONS

Do not rotate image

Do not flip image horizontally

Do not flip image vertically

COLOR TABLE:

Selected color table is |Multicolor 7 |

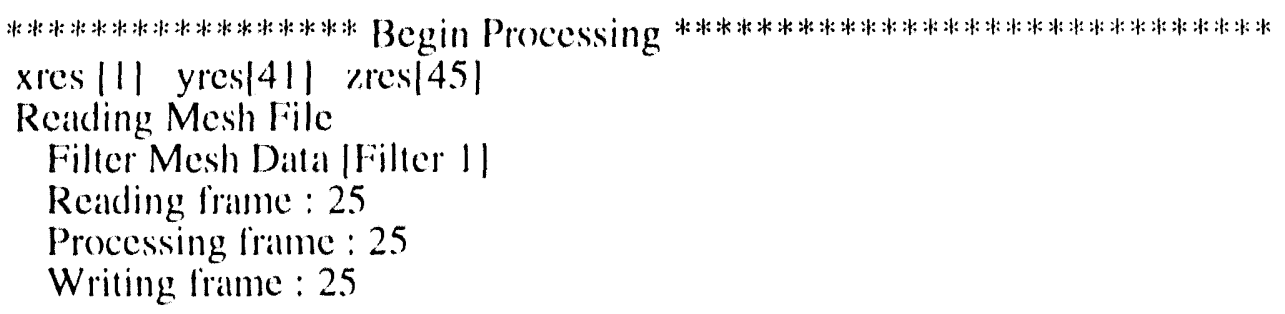

We now have the file slice01.ras, which may be displayed with xtras:

\section{xtras slice(01.ras}

This concludes our example of the TSAR EM modeling package. There are many other options available, but it is hoped that this will allow a new user to get started.

Experimenting with other selections should be less daunting now that the user has set up and run at least one TSAR problem. 

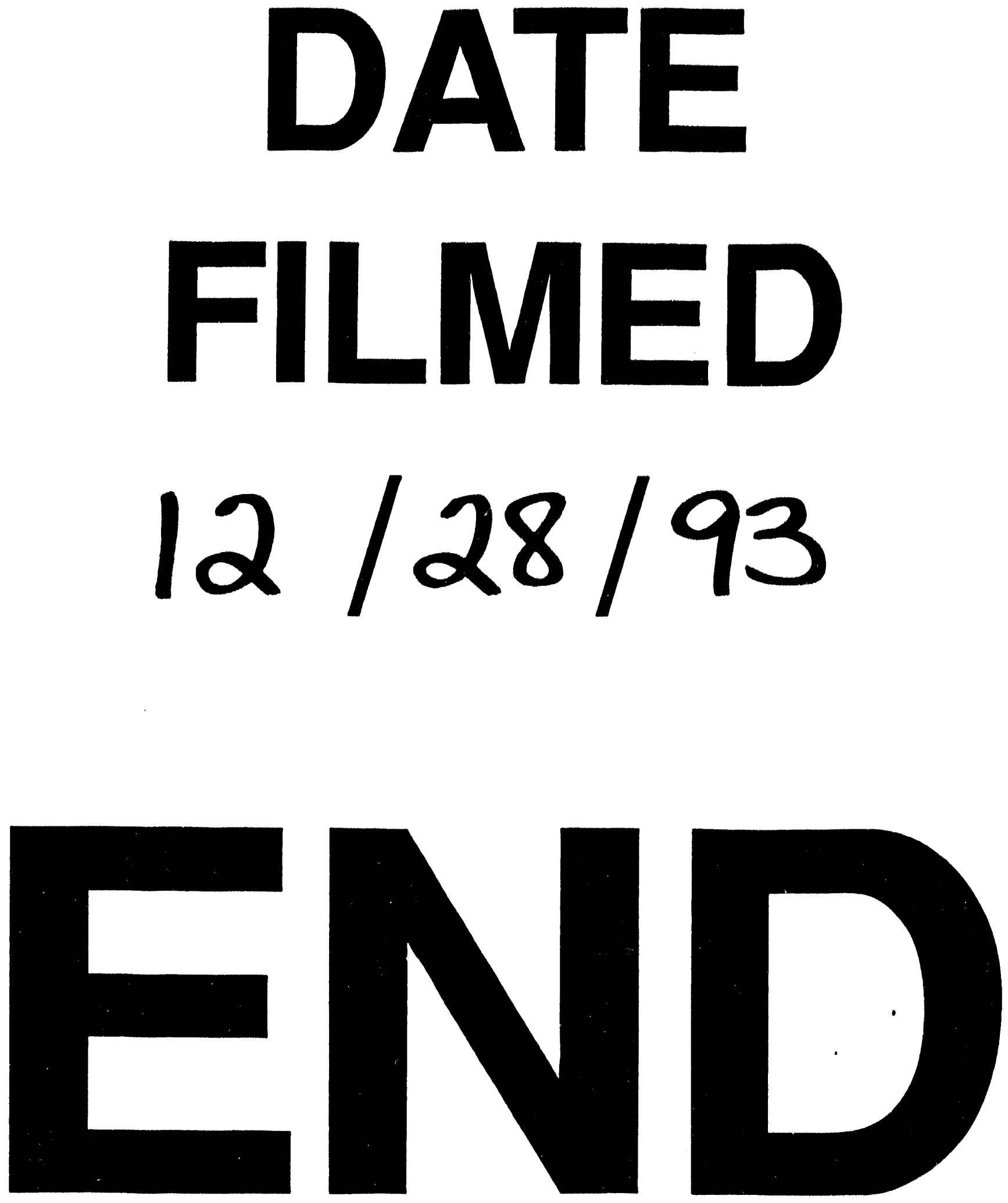

$-$ 